\title{
Anestesia inhalatoria en el equino: Características y consideraciones al momento de su uso
}

\section{Inhalation anesthesia in horses: characteristics and considerations at he time of its use}

\author{
Natalia Eckholt Peña, Camila Marañón, Felipe Corrêa* \\ Universidad Andrés Bello, Facultad de Recursos Naturales, Escuela de Medicina \\ Veterinaria \\ * Autor de correspondencia: felipe2983@gmail.com
}

Recibido: 29 de mayo de 2017; Aceptado: 10 de Octubre de 2017.

\section{RESUMEN}

Los anestésicos son fármacos potentes que no tienen un tiempo definido de uso, sin embargo, debido a las características predecibles de recuperación anestésica que poseen, y pese a que generan cambios drásticos en la función cardiovascular y respiratoria, son indispensables en medicina equina. Para lograr una anestesia balanceada combinamos el uso de diferentes fármacos endovenosos con un anestésico inhalatorio. Cual fármaco utilizar dependerá tanto del estado de salud general, como del tipo de procedimiento al que será sometido el paciente. Es por esto, que antes de someter a un paciente a anestesia, éste debe ser examinado cuidadosamente para así caracterizar su estado general. Para ello existe el sistema de clasificación ASA que permite determinar las consideraciones anestésicas y así poder disminuir los riesgos que conlleva dicho procedimiento. Es fundamental que mientras el paciente este anestesiado sea monitoreado constantemente, para así asegurar y controlar que tanto la función fisiológica, como la profundidad anestésica sean las adecuadas, lo que a su vez permitirá actuar a tiempo frente a las diversas complicaciones que se pudiesen presentar. La morbilidad y mortalidad en la anestesia equina es considerablemente mayor que en otras especies domésticas.

Palabras claves: equino; anestesia inhalatoria; protocolos; complicaciones anestésicas.

\section{SUMMARY}

Anesthetic drugs are fundamental in surgical procedures due to their flexible time of use and predictable recovery times. However, they can generate drastic changes in the cardiovascular and respiratory function of horses. To achieve a balanced anesthesia, we use several intravenous drugs with an inhalation anesthetic. The decision of which drug to use will depend on the state of the general health, as well as the kind of procedure in which the patient will undergo. Therefore, before submitting a patient under anesthesia, we must do a complete physical exam and characterize their general state. For this purpose there is the ASA classification system which allows to determinate the general condition of the patient and have the anesthetic 
considerations to lower the risk of each procedure. It is fundamental a constant monitoring of the patient while he is under anesthesia to ensure and control the physiological function and the appropriated anesthetic depth, as well as to act on time in front of the diverse complications that can present. Morbidity and mortality in equine anesthesia is considerably higher than in other domestic species.

Keywords: equine; inhalation anesthesia; protocols; anesthetic complications.

\section{INTRODUCCIÓN}

La anestesia es inducida por fármacos que deprimen la actividad del tejido nervioso local, regional o del sistema nervioso central (SNC). De este modo, trata de proporcionar inconsciencia, analgesia y relajación muscular en un individuo con el propósito de realizar procedimientos que de otra manera podrían crear estrés físico y/o psicológico. Sin embargo, los fármacos anestésicos suelen inducir efectos fisiológicos adversos que pueden conducir a un mayor riesgo de morbilidad y mortalidad en los pacientes (Tranquilli y col, 2007; Fischer, 2015). El estado anestésico "ideal" (el cual conlleva sedación, analgesia, relajación muscular y pérdida de conciencia) en caballos se logra de mejor manera administrando múltiples fármacos en combinación para producir los efectos deseados sobre la conciencia y el dolor (Muir y Hubbell, 2009). Las ventajas de este enfoque "multimodal" incluyen, un aumento en el potencial de efectos anestésicos beneficiosos aditivos o sinérgicos, un aumento en el alcance de la actividad anestésica (por ejemplo, analgesia y relajación muscular) y el potencial para reducir los efectos secundarios o un evento (Tranquilli y col, 2007; Fischer, 2015). Las desventajas incluyen el potencial de interacciones adversas de fármacos, lo que da lugar a un mayor potencial de efectos secundarios (por ejemplo, bradicardia, íleo, ataxia), acontecimientos adversos (por ejemplo, hipotensión, depresión respiratoria) y un mayor tiempo de recuperación anestésica (Muir y Hubbell, 2009).

Los agentes inhalatorios comprenden una clase indispensable de fármacos anestésicos, y en los equinos es muy utilizada para procedimientos prolongados. Son fármacos potentes que deben ser usados con precaución. Son de fácil control de administración, no tienen un límite definido de tiempo de uso y las características de recuperación predecibles los han hecho indispensables en la anestesia equina, pero el tamaño, el comportamiento y la fisiología de los caballos siguen representando importantes riesgos y desafíos para la anestesia inhalatoria en relación a otras especies (Taylor y Clarke, 2007; Brosnan, 2013).

La mortalidad perioperatoria en caballos es elevada en comparación con humanos y animales menores. Algunos estudios describen una mortalidad entre $0,6 \%$ a $1,8 \%$ y que aumenta sobre el $5 \%$ si se incluyen caballos que estén cursando con alguna alteración sistémica, mientras que en otros se observa una mortalidad de un $0.9 \%$ en equinos que son sometidos a procedimientos de diversas patologías pero que no incluyen cirugías por cólico (Mitchell, 1969; Johnston y col, 1995; Mee y col, 1998; Johnston y col, 2002). Por otra parte, se ha determinado que a mayor duración de la anestesia hay mayor mortalidad, principalmente en procedimientos sobre 2 horas y especialmente sobre las 4 horas (Johnston y col, 1995).

\section{1.- ANATOMÍA Y FISIOLOGÍA CARDIOVASCULAR}


El sistema cardiovascular consta de tres componentes, (corazón, vasos y sangre) y cuyo objetivo final es proporcionar oxígeno a las células del organismo (Doherty y Valverde, 2006), es decir, está diseñado para suministrar un flujo continuo de oxígeno y nutrientes a todos los tejidos del cuerpo (Tranquilli y col, 2007).

La entrega de oxígeno tisular $\left(\mathrm{DO}_{2}\right)$ está determinada por el producto entre la cantidad de sangre bombeada a las células (gasto cardíaco) y el contenido de oxígeno en la sangre arterial $\left(\mathrm{CaO}_{2}\right)$ (Doherty y Valverde, 2006). El funcionamiento normal de cada tejido corporal depende de la llegada de un flujo sanguíneo suficiente. De esta manera, cuanto mayor sea la actividad metabólica de un tejido, mayor será el aporte de sangre que necesita (Klein, 2014).

La anestesia puede alterar drásticamente la función cardiovascular y tener un impacto global en la función de los órganos a través de la disminución de la $\mathrm{DO}_{2}$ (Doherty y Valverde, 2006) y esto se explica principalmente ya que, si el flujo sanguíneo que llega a un tejido disminuye o es insuficiente se generará una isquemia, la cual, aunque sea transitoria, va a producir una disfunción en esos tejidos (Klein, 2014). Así mismo, se debe considerar que la absorción, distribución y eliminación de fármacos anestésicos van a depender del flujo de sangre (Tranquilli y col, 2007).

Me parece insuficiente la descripción fisiopatólogica del sistema cardiovascular y su interacción con la anestesia inhalatoria. Falta discutir en mayor profundidad componentes del $\mathrm{CaO} 2$ y el gasto cardíaco y cómo cada uno de estos parámetros se ve afectado durante el perioperatorio (p.ej, el gasto cardíaco está determinado por la frecuencia cardíaca y el débito sistólico, cada uno por separado, o en suma, se puede ver afectado por diferentes vías, p.ej, actividad parasimpática elevada o depresión miocárdica con menor débito, y en caso de $\mathrm{CaO} 2$, por alteraciones pulmonares conducentes a inadecuado intercambio de 02 , hemorragias y baja de $\mathrm{Hb}$., etc). Por otra parte, se discute de forma general los problemas anterógrados, es decir, gasto cardíaco y DO2, pero no se menciona nada acerca del retorno venoso, cómo la anestesia lo afecta y cómo puede atacarse (presión media de llene sistémico y su relación con la presión venosa central), el cual es igual o más fundamental que el gasto cardíaco. Cómo afecta el anestésico a la presión arterial?, qué pasa con la presión sistólica y diastólica?, cómo se pueden tratar las alteraciones asociadas a la presión arterial?. Por otro lado, la isquemia es el evento final de una DO2 inadecuada, pero antes de eso ocurren otros eventos, como inadecuado funcionamiento de diferentes órganos o tejidos como el muscular, y que son los que observamos en el perioperatorio, ojalá que antes de ver isquemia como tal.

\section{2.- ANATOMÍA Y FISIOLOGÍA RESPIRATORIA}

El sistema respiratorio consta de dos funciones principalmente: (1) la función respiratoria que está asociada al intercambio gaseoso y la (2) ventilación pulmonar. La ventilación es el movimiento del gas dentro y fuera de los alvéolos. En los equinos, a diferencia de otros animales, la respiración (inspiración y parte de la espiración) son procesos activos que utilizan energía metabólica, es por esto que el requisito de ventilación para la homeostasis varía con las necesidades metabólicas de los animales, y por lo tanto varía con el tamaño del cuerpo, el nivel de actividad, la temperatura corporal y en casos de procedimientos quirúrgicos, con la profundidad anestésica (Doherty y Valverde, 2006; Tranquilli y col, 2007; Klein, 2014). 
La ventilación pulmonar (alveolar) está regulada por el SNC (no utilizar abreviaciones si no se ha abreviado previamente) a través de quimiorreceptores que detectan las presiones parciales, principalmente de $\mathrm{CO}_{2} \mathrm{y}$, en menor medida, de $\mathrm{O}_{2}$ en la sangre (especialmente en condiciones en que el $\mathrm{O} 2$ está MUY bajo), y se logra mediante la expansión y relajación posterior de los pulmones (Doherty y Valverde, 2006; Tranquilli y col, 2007) (realmente se logra por la actividad diafragmática, en menor medida por la intercostal y como consecuencia por la expansión del pulmón a consecuencia de un descenso (durante ventilación espontánea) o un aumento (durante ventilación positiva) en la presión pleural con respecto a la presión atmosférica.

Los animales cubren su demanda metabólica de oxígeno inspirando un cierto volumen de aire cada minuto. El volumen de cada respiración (o volumen tidal (VT) y la frecuencia respiratoria ( $f$ ) determinan el volumen de aire respirado por minuto, que se conoce como ventilación minuto $\left(V_{E}\right)$ y este $V_{E}$ puede sufrir cambios por necesidad del metabolismo y que se pueden realizar mediante modificaciones en el VT, la frecuencia respiratoria o ambos (Muir y Hubbell, 2009; Klein, 2014).

El aire va a fluir hasta los alvéolos atravesando las diferentes estructuras anatómicas que forman parte de las vías respiratorias conductoras, pero el intercambio gaseoso no ocurre en ellas, es por esto que se les denomina como espacio muerto anatómico (Klein, 2014). También puede existir la presencia de espacio muerto en los alvéolos, que se produce por una irrigación alveolar deficiente la cual va a impedir un intercambio gaseoso adecuado, a este se le denomina espacio muerto alveolar. La suma del espacio muerto anatómico y espacio muerto alveolar da como resultado lo que se conoce como espacio muerto fisiológico (Doherty y Valverde, 2006; Muir y Hubbell, 2009; Klein, 2014).

Una porción de cada VT, y por lo tanto de ventilación alveolar (VA), ventila el espacio muerto anatómico (VD), y una porción de VA participa en el intercambio gaseoso. La ventilación alveolar (VA) está regulado por mecanismos de control para adaptarse a la absorción de oxígeno y la eliminación de dióxido de carbono necesaria por el metabolismo. Por tanto, cuando un animal realiza ejercicio, su ventilación alveolar aumenta para captar más $\mathrm{O}_{2}$ y eliminar más $\mathrm{CO}_{2}$. La relación ventilación espacio muerto (VD)/Volumen tidal (VT) es muy variable entre las especies, y oscila entre un 50 a $75 \%$ en equinos, VD corresponde a la fracción de cada respiración que ventila este espacio muerto. Esta medición se basa en el hecho de que el $\mathrm{CO}_{2}$ expirado proviene de los alvéolos perfundidos y nada desde los espacios muertos (Doherty y Valverde, 2006; Muir y Hubbell, 2009; Klein, 2014).

Finalmente, es importante conocer la relación ventilación/perfusión (V/Q) que presenta el equino. En un caballo normal, la relación $\mathrm{V} / \mathrm{Q}$ es cercana a 1,0, pero esta relación puede verse alterada por la distribución de la ventilación, la perfusión y/o un cambio en su distribución relativa (Doherty y Valverde, 2006). Si tenemos una unidad de pulmón que presenta poco o nada de ventilación con respecto a la perfusión, la sangre que sale de esta unidad tendrá un contenido de $\mathrm{O} 2$ menor que las unidades con relaciones $\mathrm{V} / \mathrm{Q}$ óptimas. Si tenemos unidades con una relación $\mathrm{V} / \mathrm{Q}=0$, la sangre que sale de esta unidad tendrá un contenido de $\mathrm{O}_{2}$ similar a la sangre de la arteria pulmonar. En esta última situación, la sangre que sale de esta unidad se conoce como una especie de cortocircuito intrapulmonar y comúnmente se da como resultado de atelectasia, o por una obstrucción parcial o completa de las vías respiratorias (Doherty 
y Valverde, 2006). La importancia de conocer la fisiología del sistema respiratorio radica en que, al momento de someter a un paciente a cualquier tipo de sedación o anestesia, estaremos generando cambios en su funcionamiento (Doherty y Valverde, 2006; Muir y Hubbell, 2009).

\section{3.- FÁRMACOS COMUNMENTE UTILIZADOS EN ANESTESIA EQUINA}

Existen diferentes tipos de métodos anestésicos y la elección de cual utilizar está asociado al tipo de procedimiento al que debe ser sometido el paciente. Para lograr una anestesia balanceada es que combinamos el uso de diferentes fármacos endovenosos con un anestésico inhalatorio.

\section{1.- Fármacos inyectables}

3.1.1.- Ketamina: anestésico disociativo ampliamente utilizado como agente de inducción en equinos. Se reconoce como un antagonista del receptor NMDA y, a dosis muy bajas, puede contribuir sustancialmente a la analgesia minimizando la sensibilización al sistema nervioso central. Es metabolizada en el hígado y excretada por los riñones. No se debe utilizar sólo como anestésico en los equinos debido a la incoordinación muscular que genera, además del peligro potencial por un comportamiento incontrolable del paciente. Este fármaco, además de poder brindar variables grados de analgesia, se caracteriza por producir catalepsia y una pobre relajación muscular. Se administra por lo tanto en combinación con sedantes hipnóticos, relajantes musculares o analgésicos. Con estas combinaciones se produce anestesia de corta duración que puede ser usada para procedimientos cortos o como inductor para la anestesia inhalatoria (Doherty y Valverde, 2006; Tranquilli y col, 2007; Muir y Hubbell, 2009; Lin y Col, 2015).

3.1.2.- Xilacina: corresponde a un agonista a 2 adrenérgico, y debido a sus efectos sedantes y analgésicos potentes, los a2 agonistas son de gran importancia en la anestesia equina. Actúa como coadyuvante anestésico y ayuda a eliminar la hipertonicidad muscular que se genera por el uso de ketamina. Produce una sedación rápida, predecible, además de analgesia y relajación muscular después de su administración endovenosa. En los caballos, el punto más alto de sedación y analgesia se desarrolla, dependiendo de la dosis, en 5 - 10 minutos y su efecto persiste durante los siguientes 20 - 30 minutos. Como punto importante, cabe destacar que la administración prequirúrgica de xilacina reduce los requerimientos (concentración alveolar mínima o CAM) de halotano e isoflorano en animales (Doherty y Valverde, 2006; Tranquilli y col, 2007; Muir y Hubbell, 2009).

3.1.3.- Acepromacina: se clasifica como un tranquilizante y es la fenotiazina más comúnmente utilizada. Ejercen su efecto tranquilizante al bloquear los receptores de dopamina. Produce vasodilatación que conlleva a una hipotensión. Su uso ayuda a reducir los requerimientos de anestesia inhalatoria (CAM). Se demora aproximadamente 20 minutos en hacer efecto después de la administración vía endovenosa. No tiene acción analgésica y para obtener una sedación más fiable se recomienda su combinación con opioides o un agonista a2 adrenérgico. Su uso está asociado a una reducción en el riesgo de muerte intraquirúrgico, aunque no está completamente clara cuál sería la razón, pero uno de los motivos podría ser que reduce la poscarga (Doherty y Valverde, 2006; Tranquilli y col, 2007; Muir y Hubbell, 2009). 
3.1.4.- Diazepam: este fármaco corresponde a una Benzodiacepina. No está indicado su uso para procedimientos en que el paciente deba mantenerse en pie ya que genera relajación muscular y ataxia, en potrillos neonatos esta combinación debe ser administrada lentamente ya que pueden causar una depresión respiratoria. Este fármaco es metabolizado principalmente por el hígado y excretado por la orina. No se debe administrar vía IM ya que es irritante y tiene poca absorción. Tiene una vida media larga. Insoluble en agua y presenta metabolitos activos. Su uso en conjunto con ketamina reduce la dosis necesaria de benzodiacepina (Doherty y Valverde, 2006; Tranquilli y col, 2007; Muir y Hubbell, 2009).

3.1.5. - Midazolam: fármaco de la familia de las benzodiacepinas y que se utiliza para lograr relajación muscular durante la inducción anestésica. Soluble en agua, tiene una vida media menor a la del diazepam y no cuenta con metabolitos activos. Al igual que diazepam, no está indicado su uso para procedimientos en que el paciente deba mantenerse de pie (Doherty y Valverde, 2006; Tranquilli y col, 2007; Muir y Hubbell, 2009).

3.1.6.- Lidocaína: anestésico local más utilizado en la práctica clínica debido a su potencia, es de inicio rápido (5 a 10 minutos), presenta una duración de acción moderada (60-90 minutos) y actividad anestésica tópica. Es altamente metabolizada en el hígado y su clearance depende de las tasas del flujo sanguíneo hepático, el cual se ha visto que disminuye durante la anestesia inhalatoria. También se puede administrar por vía endovenosa mediante infusión ya que ayuda a disminuir los requerimientos anestésicos inhalatorios (CAM), llegando a reducir la CAM entre un 25 a un $50 \%$ según la dosis utilizada. Además, se ha demostrado que favorece la motilidad intestinal cuando se administra como infusión durante la cirugía de cólicos, proporciona analgesia durante el posoperatorio y no se ha evidenciado que pueda inducir efectos cardiovasculares significativos. Sin embargo, se ha demostrado que su uso aumenta la presentación de ataxia en los pacientes en recuperación anestésica, por lo que, se ha estimado que la infusión debe detenerse 30 minutos antes de finalizar el tiempo anestésico (Doherty y Valverde, 2006; Taylor y Clarke, 2007; Muir y Hubbell, 2009).

\section{2. - Fármacos inhalatorios}

Los anestésicos inhalatorios son un grupo de fármacos que, por sí mismos o en combinación con otros tipos de fármacos, producen anestesia general. En los equinos, la anestesia en procedimientos prolongados se mantiene generalmente por inhalación de un agente anestésico volátil. Son fármacos potentes, por lo cual deben ser usados con precaución. Sin embargo, son de fácil control en la administración de estos, además de no existir un límite definido a la duración del uso y posee características de recuperación predecibles, por lo que las han hecho indispensables en la anestesia equina (Taylor y Clarke, 2007). Se absorben en la sangre a través de la circulación pulmonar, en donde depende principalmente de la ventilación alveolar, la solubilidad de la droga inhalada, el gasto cardíaco y la diferencia de presión parcial del anestésico inhalatorio entre la sangre venosa y los alvéolos, luego son llevados al cerebro, donde causan anestesia. La profundidad de la anestesia es proporcional a la presión parcial que ejercen en el cerebro (órgano target) y no a la masa de la droga que circula en el cuerpo. Dentro de las desventajas, los anestésicos inhalatorios producen depresión cardiorrespiratoria, la cual está relacionada con la dosis que se usan, y esto resulta en 
una disminución del gasto cardíaco, de la presión sanguínea arterial (por vasodilatación), de la perfusión tisular y de la oxigenación tisular (Doherty y Valverde, 2006; Taylor y Clarke, 2007; Muir y Hubbell, 2009).

La concentración alveolar mínima o CAM, corresponde a la concentración (a presión atmosférica) que impide que el $50 \%$ de los animales se muevan en respuesta a un estímulo nocivo, por lo tanto, es una medida de la potencia que tienen los agentes anestésicos inhalatorios. Por otra parte, tenemos que la profundidad anestésica está relacionada con la presión parcial del anestésico a nivel cerebral y esta tensión cerebral es proporcional a la concentración alveolar, por lo cual, se usa la concentración alveolar (tomada como concentración del end-tidal y posible de monitorizar) como medida de la profundidad de la anestesia (Taylor y Clarke, 2007; Muir y Hubbell, 2009). Se ha determinado que si se utiliza solo un gas inhalatorio como método anestésico, la CAM necesaria es de aproximadamente 1.5 para una cirugía. Sin embargo, actualmente, los agentes inhalatorios rara vez se usan solos, hoy en día se usa el concepto de anestesia balanceada, en donde el uso simultáneo de sedantes, anestésicos inyectables y analgésicos, así como la analgesia mediante anestesia local proporciona una mejor transición y mantenimiento de la anestesia, reduce los requerimientos de inhalantes y mejora la función cardiovascular en caballos al reducir la dosis requerida del agente inhalatorio y por ende la CAM (Taylor y Clarke, 2007; Muir y Hubbell, 2009).

Según Doherty y Valverde (2006), para poder evitar el movimiento durante los procedimientos quirúrgicos, generalmente es necesario mantener las concentraciones del end-tidal en aproximadamente un 30\% más que la CAM. Además, existen diversos factores y fármacos que pueden generar cambios en los valores de CAM en un paciente. Los factores que causan un aumento de la CAM son: 1) Estimulantes del Sistema nervioso central (SNC), 2) Edad (de qué manera lo aumenta, joven o viejo) y 3) Hipertermia. Por otra parte, los factores que generan una disminución de la CAM son: 1) Hipotermia, 2) Edad (extremos, pacientes neonatos y seniors), 3) Sedantes y analgésicos, 4) Preñez y 5) Endotoxemia. Existen fármacos que también generan una disminución de la CAM, dentro de estos podemos encontrar: Acepromacina, a2 agonistas (xilacina, detomidina, etc.), ketamina, benzodiacepinas (diazepam, Midazolam, etc.) y lidocaína.

3.2.1.- Isoflurano: agente inhalatorio más utilizado en anestesia equina, se caracteriza por no ser inflamable y se vaporiza fácilmente, además tiene menor solubilidad en sangre y grasa. Solo una pequeña fracción se metaboliza en el hígado, ya que gran parte es exhalada sin sufrir cambios. Sus propiedades físicas permiten una rápida inducción y estabilización de la anestesia, pero también es más fácil dar una sobredosis al paciente. La CAM del isoflorano es cercano al 1,2\%, cuando es asociado a una premedicación para la inducción, por lo cual los requerimientos suficientes de este agente en una cirugía serán entre un 1.3\% a 1.5\%. La recuperación de la anestesia con isoflorano en ocasiones se asocia con desorientación y un grado de violencia, por lo que se debe reducir el riesgo de esto mediante el uso de sedación. Dentro de las desventajas que presenta podemos encontrar los efectos que genera en el sistema cardiovascular y respiratorio. Genera aumentos en la frecuencia cardiaca, vasodilatación periférica y, por lo tanto, hipotensión, que puede llegar a ser grave si no se controla. También se describe que si se usa a niveles alveolares cercanos a la CAM 
ocurre poca depresión miocárdica, por lo que el flujo sanguíneo periférico es bueno. La depresión miocárdica ocurre en dosis más altas. Se caracteriza por ser un potente depresor respiratorio, en ocasiones la depresión respiratoria a menudo es tal que retarda la absorción de isoflorano, por lo que puede ser difícil obtener una anestesia adecuada. Diferentes estudios indican que, en comparación con el halotano, posee un mayor margen de seguridad cardiovascular, es decir, tendría menores efectos cardiovasculares (Raisis y Col, 2000; Johnston, 2004; Doherty y Valverde, 2006; Taylor y Clarke, 2007; Tranquilli y col, 2007; Muir y Hubbell, 2009; Brosnan, 2013).

3.2.2.- Sevoflorano: agente volátil potente, con una CAM de aproximadamente $2,8 \%$, no es irritante. Concentraciones de end-tidal de $2.8-3.0 \%$ son generalmente adecuadas para mantener una anestesia estable. Tiene una menor solubilidad en sangre y grasa que el isoflorano, por lo que la inducción y la recuperación de la anestesia son muy rápidas y es fácil mantener una profundidad anestésica estable. La rápida absorción del sevoflorano hace que sea fácil de sobredosificar. Tiene efectos cardiovasculares y respiratorios similares a los del isoflorano. La hipotensión resulta de la vasodilatación, y la función cardíaca se mantiene bien. Genera una recuperación rápida $y$, aunque se cree que es más calmada que la del isoflorano, la rapidez puede conducir a la desorientación y el paciente puede volverse violento. Se debe usar con consideración y de forma juiciosa la sedación que reducirá o evitará recuperaciones de mala calidad (Doherty y Valverde, 2006; Taylor y Clarke, 2007; Tranquilli y col, 2007; Muir y Hubbell, 2009; Brosnan, 2013).

3.2.3.- Halotano: fue utilizado por primera vez en la anestesia equina en el año 1957, logrando una anestesia prolongada seguida de una recuperación suave y predecible. Es un potente anestésico que no irrita el tracto respiratorio, no es inflamable, pero es sensible a la luz. Es más soluble en sangre y grasa en comparación al isoflorano, es por esto que la inducción y la recuperación son un poco más lentas. Estudios epidemiológicos han demostrado que para la anestesia equina la seguridad general del halotano e isoflorano son iguales. Sin embargo, la principal desventaja del halotano en equinos es el grado de depresión que genera en el miocardio. Sus ventajas son: la capacidad de mantener respiración espontánea, y que en la mayoría de los casos se obtiene una recuperación suave y tranquila, por lo que no requiere el uso complementario de sedantes. Su CAM es cercano al $0.8 \%$, lo que significa que una concentración alveolar de $0.8-1.2 \%$ debe ser suficiente para mantener la anestesia para una cirugía, previa premedicación para la inducción (Doherty y Valverde, 2006; Taylor y Clarke, 2007; Tranquilli y col, 2007; Muir y Hubbell, 2009). Es un potente depresor miocárdico, que conduce a disminuciones en el gasto cardíaco, el cual generalmente disminuye debido a una baja en el volumen sistólico, y por ende genera hipotensión. Como todos los anestésicos inhalatorios, causa depresión respiratoria al reducir la respuesta al dióxido de carbono, la frecuencia respiratoria disminuye, observándose un aumento de la presión parcial de $\mathrm{CO}_{2}$ y una disminución de la capacidad de oxigenación de la sangre arterial. Sin embargo, la depresión respiratoria es menor que la del isoflorano. Al igual que los otros agentes anestésicos inhalatorios tiene muy poca metabolización hepática, ya que gran parte se elimina por la espiración (Raisis y Col, 2000; Johnston, 2004; Doherty y Valverde, 2006; Taylor y Clarke, 2007; Tranquilli y col, 2007; Muir y Hubbell, 2009; Brosnan, 2013). 
3,2,4.- Oxígeno: todo anestésico inhalatorio debe ir acompañado de un "gas portador" y este debe contener un mínimo de 50\% de oxígeno, e incluso usualmente, es más. El oxígeno es suministrado al circuito de respiración desde una fuente de baja presión en la máquina anestésica y se suministra al circuito respiratorio a una velocidad de flujo adecuado para transportar suficiente agente anestésico y para proporcionar los requerimientos metabólicos de oxígeno necesarios. Los medidores de flujo dirigen el flujo de $\mathrm{O}_{2}$ desde el regulador al vaporizador. El flujo de gas fresco se ajusta lo suficiente para mantener la bolsa reservorio inflada (Doherty y Valverde, 2006; Taylor y Clarke, 2007; Muir y Hubbell, 2009).

\section{4.- EQUIPO DE ANESTESIA Y PREPARACIÓN DEL PACIENTE}

4.1.- Equipo de anestesia

En el mercado existen diversos equipos de anestesia. La mayoría de las máquinas incorporan un sistema circular con un vaporizador fuera del círculo. Algunas máquinas vienen equipadas con un ventilador mecánico. La máquina anestésica y el circuito respiratorio están diseñados para facilitar el suministro controlado de oxígeno y gases anestésicos, y eliminar el dióxido de carbono del gas exhalado. Son diferentes componentes los que forman parte del equipo de anestesia, todos los cuales deben ser revisados antes de entrar a un paciente a un procedimiento bajo anestesia general. Entre los componentes encontramos (Doherty y Valverde, 2006; Taylor y Clarke, 2007; Muir y Hubbell, 2009; Moens, 2013):

Medidores de flujo: hay uno para cada tipo de gas disponible. Este medidor controla y muestra la velocidad (L/min) del flujo de gas. Es una válvula ajustada por un botón de control con color y táctil; un tubo de vidrio cónico con números que corresponden a velocidades de flujo de gas y un flotador, que se eleva dentro del tubo de vidrio para indicar el flujo de gas que se está administrando. Cuanto más gas se permite entrar, más alto sube el flotador dentro del tubo (Doherty y Valverde, 2006; Taylor y Clarke, 2007; Muir y Hubbell, 2009; Moens, 2013).

Válvula de descarga de oxígeno: esta válvula suministra oxígeno directamente en el circuito de respiración a flujos relativamente altos. El oxígeno evita el vaporizador anestésico. La mayoría de las válvulas de descarga de oxígeno entregan el oxígeno entre 35 y 75 L/min. La activación de la válvula de descarga de oxígeno llenará el circuito anestésico con $100 \%$ de oxígeno y diluirá la concentración de gas anestésico (Doherty y Valverde, 2006; Taylor y Clarke, 2007; Muir y Hubbell, 2009; Moens, 2013).

Vaporizadores: convierte el anestésico de inhalación de líquido volátil a vapor. Los vaporizadores de precisión están diseñados para proporcionar una concentración constante de agente anestésico en cada ajuste a pesar de los cambios en flujo y temperatura. El oxígeno se pasa desde el medidor de flujo al vaporizador que contiene el líquido anestésico y se satura con el anestésico. El gas lleva así el anestésico, ahora vaporizado, al circuito respiratorio. Las funciones del vaporizador son almacenar el anestésico líquido y entregar el volumen deseado del vapor anestésico al flujo de gas fresco (Doherty y Valverde, 2006; Taylor y Clarke, 2007; Muir y Hubbell, 2009; Moens, 2013).

Salida de gas común: suministra gas desde los medidores de flujo y vaporizadores al circuito de respiración anestésica. Presenta un tubo que está conectado al circuito 
respiratorio anestésico y suministra el gas portador fresco (es decir, el oxígeno) y el vapor anestésico (Doherty y Valverde, 2006; Taylor y Clarke, 2007; Muir y Hubbell, 2009).

Sistemas o Circuitos anestésicos: la función de un circuito es suministrar oxígeno y anestésico, además de eliminar $\mathrm{CO}_{2}$. El circuito se coloca entre la salida de gas que se encuentra en la máquina anestésica y el traqueotubo en el caballo. Existen diferentes sistemas disponibles, pero los habitualmente utilizado son: Los circuitos cerrados o circulares que preferentemente son utilizados en caballos y los circuitos abiertos o de ida y vuelta que se usan menos comúnmente. En el circuito abierto el paciente recibe directamente los gases provenientes desde el equipo de anestesia. Por otra parte, en el circuito cerrado todo el gas espirado es reinhalado, y al sistema se va incorporando el $\mathrm{O}_{2}$ necesario y el agente anestésico inhalatorio. El circuito cerrado está compuesto de: colector (canister) de absorción de dióxido de carbono, válvula de alivio de presión (pop-off) y una bolsa reservorio. Además, también utiliza válvulas unidireccionales, una pieza en $\mathrm{Y}$ y mangueras de respiración corrugadas (una de inspiración y la otra de espiración) (Doherty y Valverde, 2006; Taylor y Clarke, 2007; Muir y Hubbell, 2009).

Canister: recipiente que contiene generalmente cal sodada la cual absorbe el $\mathrm{CO}_{2}$ que proviene desde el tubo de espiración. El material absorbente elimina el dióxido de carbono del gas exhalado y en el proceso genera calor y humedad. El cambio de color del material absorbente es indicativo del "agotamiento" de este y por ende ya dejará de cumplir su función adecuadamente. Este cambio de color es mayor en la entrada del recipiente y se extiende desde el centro hasta las paredes, donde la resistencia al flujo de gas es menor. La duración y la eficacia del material absorbente antes de su modificación varía con el tamaño del caballo, la producción individual de CO2 (tasa metabólica), el flujo de gas fresco, el tamaño y la ubicación del recipiente en relación con las vías respiratorias del paciente y otros componentes del circuito respiratorio. Cuanto mayor sea el cambio de color, más completo será el agotamiento (Doherty y Valverde, 2006; Taylor y Clarke, 2007; Muir y Hubbell, 2009).

Bolsa de reservorio: proporciona un margen para cambiar los volúmenes dentro del sistema respiratorio y un medio para ayudar o controlar la respiración. Además, proporciona una indicación visual de la ventilación espontánea del paciente (Doherty y Valverde, 2006; Taylor y Clarke, 2007; Muir y Hubbell, 2009).

Válvula de alivio de presión (pop-off): está diseñada para ventilar el gas del circuito de respiración y controlar la presión dentro. Cuanto mayor sea el flujo de gas fresco, habrá más escapes de gas a través de la válvula pop-off. La válvula se deja en posición abierta en todo momento cuando los flujos de gas fresco exceden el consumo de oxígeno del paciente, excepto durante los períodos de ventilación asistida o mecánica en donde hay una válvula de alivio de presión que está integrada, esta válvula se abre automáticamente en espiración y "cierra" en inspiración, durante el ciclo respiratorio mecánico. Estas válvulas de alivio de presión frecuentemente están equipadas con un dispositivo de recogida que dirige gas residual a un sistema de eliminación de gas a través de una manguera flexible ondulada (Doherty y Valverde, 2006; Taylor y Clarke, 2007; Muir y Hubbell, 2009; Moens, 2013).

Mangueras de Respiración: están construidas de plástico ligero o de caucho, son corrugadas para evitar la torcedura y el flujo turbulento. Estas mangueras son iguales en diámetro o incluso más grandes que la tráquea del caballo. Los tubos de mayor 
diámetro ofrecen menos resistencia que los tubos de menor diámetro (Doherty y Valverde, 2006; Taylor y Clarke, 2007; Muir y Hubbell, 2009; Moens, 2013).

Conector de Pieza Y: esta pieza conecta las mangueras inspiratoria y espiratoria del circuito anestésico circular a una máscara o tubo endotraqueal usando un adaptador de plástico o metálico. Alternativamente, algunos tubos endotraqueales tienen un adaptador flexible que se desliza sobre la pieza Y (Doherty y Valverde, 2006; Taylor y Clarke, 2007; Muir y Hubbell, 2009; Moens, 2013).

Flujo de gases frescos: este flujo de gas es el transportador del anestésico inhalatorio. Generalmente se usan flujos de gas fresco relativamente bajos, lo que da lugar a la conservación de gases anestésicos. Un flujo de oxígeno que iguala el consumo mínimo de oxígeno del equino, minimiza la cantidad de gas anestésico utilizado y la cantidad eliminada como residuo. La desventaja del oxígeno a bajo flujo es que los flujos de gas fresco relativamente bajos y un vaporizador convencional pueden retrasar o impedir la obtención de una concentración anestésica suficiente dentro del sistema para inducir la anestesia. Además, se debe considerar, que estas desventajas en parte se relacionan con el volumen relativamente grande del circuito anestésico y del volumen pulmonar de los equinos. El flujo de oxígeno para un sistema de circuito anestésico de círculo equino debe ajustarse a unos 8 a $12 \mathrm{Lt} / \mathrm{min}$ durante los primeros 10 a 15 minutos después de la inducción a la anestesia (Doherty y Valverde, 2006; Taylor y Clarke, 2007; Muir y Hubbell, 2009; Moens, 2013).

Ventilador mecánico: La ventilación mecánica en caballos se ha documentado desde principios del siglo XIX. La mayoría de los equipos anestésicos actuales que se utilizan en equinos adultos vienen con ventilador incorporado. Estos ventiladores son accionados por energía eléctrica, gas comprimido, o ambos. Para conectar el ventilador en la mayoría de los sistemas, se retira la bolsa reservorio y se completa el circuito uniendo el soporte de la bolsa reservorio a un fuelle (con una longitud de tubería) alojado dentro de una cámara de plástico. La cámara se presuriza con un gas impulsor, que puede ser $\mathrm{O}_{2}$ o aire comprimido, haciendo que el fuelle se vacíe. El gas impulsor sale de la cámara durante la espiración. Cualquier ventilador generalmente permite regular tanto el volumen como la presión y debe tener un dispositivo limitador de la presión para prevenir el barotrauma en los pulmones del paciente anestesiado. La ventilación mecánica de los caballos durante la anestesia sigue siendo una herramienta crucial para el manejo anestésico óptimo en estas especies propensas a hipercapnia e hipoxemia si se producen los posibles efectos cardiovasculares secundarios (Doherty y Valverde, 2006; Taylor y Clarke, 2007; Muir y Hubbell, 2009; Moens, 2013).

\section{2.- Tubos endotraqueales.}

La intubación traqueal es el método más seguro para mantener una vía aérea permeable y minimizar la aspiración. Los tubos traqueales utilizados para caballos son relativamente largos y de gran diámetro. Los tubos traqueales están disponibles en una variedad de diámetros y el tamaño del tubo se indica mediante el diámetro interno o externo en milímetros (Muir y Hubbell, 2009).

Siempre se debe tener en consideración tener mínimo 2 tubos disponibles para realizar la intubación del paciente, siendo uno del tamaño previsto y otro más chico, en caso de problemas al momento de instaurar la vía aérea. El cuff del tubo lo que hace es sellar el espacio que queda entre el tubo traqueal y la pared traqueal, ayudando a 
minimizar o prevenir la aspiración de líquido y la fuga de aire y gases anestésicos alrededor del tubo. Una presión de contacto entre la pared traqueal y el cuff de entre 20 y $30 \mathrm{~cm}$ de $\mathrm{H}_{2} \mathrm{O}$ es adecuada para sellar la vía aérea y prevenir el daño de la mucosa. Se debe revisar el cuff de los tubos seleccionados antes de utilizarlos, así verificar que estos no presenten fugas, lo indicado es dejarlo inflado unos 5 a 10 minutos para corroborar que no exista una fuga lenta (Taylor y Clarke, 2007; Muir y Hubbell, 2009). Es de gran relevancia la verificación del estado de los tubos endotraqueales, sobre todo si se utilizara en pacientes bajo ventilación mecánica o en pacientes que entran a cirugía por cólico, ya que pueden presentar regurgitación durante la anestesia, y la aspiración de contenido intestinal, sangre o soluciones quirúrgicas puede conducir a complicaciones respiratorias fatales. En algunos casos se requiere la colocación de un tubo nasotraqueal durante la recuperación anestésica, así se busca minimizar o evitar la obstrucción de la vía aérea superior durante el período de recuperación (Taylor y Clarke, 2007; Muir y Hubbell, 2009). El mantenimiento de una vía aérea permeable es el objetivo principal de toda anestesia.

\section{3.- Cateterización.}

Siempre se debe colocar un catéter endovenoso, ya sea para un procedimiento corto en terreno o sobre todo al someter a un paciente a anestesia general mantenido por anestesia inhalatoria. Estos catéteres vasculares se ponen en venas o arterias para proporcionar acceso rápido a los vasos sanguíneos para: 1) administrar fluidos, (2) obtener muestras de sangre, (3) monitorear la presión arterial, o (4) administrar fármacos. También proporciona un acceso venoso listo si deben administrarse medicamentos en casos de emergencia o fármacos de rescate. En los procedimientos quirúrgicos el acceso endovenoso (IV) es fundamental para la administración constante de fluidos, para medicamentos adicionales y para los anestésicos IV que se utilizan como complemento como por ejemplo para ayudar en el proceso de recuperación anestésica (Taylor y Clarke, 2007). El uso de un catéter nos evita el desperdicio de fármacos y la necrosis tisular por inyección perivascular inadvertida cuando se administran fármacos tóxicos o irritantes de tejido (por ejemplo, tiopental). Dentro de las venas que se pueden utilizar para instaurar una vía venosa en el equino encontramos: 1) Vena Yugular; 2) Vena cefálica y 3) Vena Safena, también está descrito que se pueden utilizar las venas torácicas lateral y medial. De todas estas, la más utilizada siempre, por su fácil acceso es la vena yugular, la cual se caracteriza porque: son fácilmente visualizables y palpables muy accesibles, grandes en diámetro y tienen un flujo sanguíneo relativamente rápido, lo que disminuye el riesgo de trombosis. La instauración de un catéter debe ir acompañado de una limpieza previa en la zona donde será puesto el catéter y de la posterior fijación adecuada, para no perder el acceso venoso. Pueden existir complicaciones asociadas al uso de un catéter endovenoso y que se deben tener en consideración, como por ej.: Tromboflebitis, Embolismo gaseoso (aire), trombosis, sangramiento excesivo, hematoma y rotura del catéter, entre otras. (Doherty y Valverde, 2006; Taylor y Clarke, 2007; Muir y Hubbell, 2009). 
4.4.- Sala de derribo del paciente sometido a anestesia inhalatoria.

El entorno en el que se lleva a cabo el procedimiento, desde la inducción hasta la recuperación, debe ser apropiado, en un ambiente seguro y controlado. Al momento de la inducción anestésica, el paciente presentara desorientación, incoordinación hasta caer y una posterior sedación, momento en el cual se intuba al paciente y se termina la preparación para ingresar a pabellón, es por esto, que la sala de derribo donde se realiza la inducción anestésica debe tener piso y paredes acolchadas y estas deben ser lo suficientemente sólidas por la presión o fuerza que es capaz de ejercer un caballo sobre estas estructuras. Aunque no hay un consenso sobre el tamaño y forma que deben tener, generalmente se considera que una sala de entre $3 \times 3$ a 4 × 4 metros es lo suficientemente amplia para realizar el derribo como para la posterior recuperación anestésica del equino. Estas salas se ubican continuas al pabellón por seguridad y para facilitar el traslado del equino anestesiado. Las habitaciones de gran tamaño no se recomiendan, ya que no proporcionan ninguna ventaja, por el contrario, al ser más espaciosas pueden permitir que el caballo pueda tomar cierta velocidad al incorporarse y desplazarse y por ende así lesionarse por un exceso de impulso que lo lleve a un impacto más fuerte contra una pared (Doherty y Valverde, 2006; Taylor y Clarke, 2007; Muir y Hubbell, 2009; Clark-Price, 2013). La superficie del piso debe proporcionar amortiguamiento por si el caballo cae, además de ser un suelo blando e idealmente con una superficie antideslizante, generalmente se debe contar con colchón de espuma móvil de unos 30 a $40 \mathrm{~cm}$ de espesor. Las paredes deben ser de al menos 2.5 metros de alto y siempre deben ser acolchadas. La sala debe contar con anillos metálicos que sean capaces de soportar fuerzas sobre $1.000 \mathrm{~kg}$ y deben estar fijados en al menos 3 paredes en los puntos más altos de la habitación. Estos anillos permiten el paso de cuerdas que ayuden a la sujeción del animal para ayudar a sostenerlo y a incorporarse. Debe haber una zona de observación, ya sea por medio de una ventana en una puerta que permita vigilar o mediante cámaras o una plataforma de observación que permita ayudar y supervisar al paciente sobre todo al momento de la recuperación. Las puertas de acceso deben ser bloqueadas por barras transversales. Además, las puertas o muros deben tener zonas despejadas en lo alto que permita el paso de las cuerdas hacia el exterior de la sala de derribo y recuperación, para así permitir el manejo de estas desde fuera. Idealmente debería contar con un sistema de calefacción y aire acondicionado para tener un control climático de la habitación según las necesidades del paciente anestesiado. En el techo, al centro de la sala se deja instalado un monorriel por el cual mediante ganchos se amarra y traslada al paciente anestesiado hacia la mesa quirúrgica de pabellón y luego de pabellón a la sala de recuperación y derribo. Si bien, estas salas en su mayoría se hacen cuadradas o rectangulares, esta descrito que lo ideal sería una habitación de forma octagonal o redonda ya que reducen la posibilidad de que un paciente quede atrapado en una esquina por desorientación e incoordinación. Finalmente, es importante que estas salas cuenten con vías de escape o salidas alternativas y rápidas para el personal (Doherty y Valverde, 2006; Taylor y Clarke, 2007; Muir y Hubbell, 2009; Clark-Price, 2013).

\section{5.- CONSIDERACIONES Y RIESGOS ANESTÉSICOS}

Aunque el riesgo anestésico, el riesgo quirúrgico y el estado físico del caballo están relacionados entre sí, no son lo mismo. El conocimiento y la habilidad del anestesista, 
el tipo de fármacos anestésicos utilizados, así como la duración de la anestesia son factores a considerar para determinar el riesgo anestésico. En general, existe una correlación entre el estado físico del caballo y aumento de la morbilidad y la mortalidad (Muir y Hubbell, 2009).

La anestesia general conlleva un riesgo de muerte o accidente grave en cualquier especie, pero el riesgo de mortalidad o morbilidad grave es particularmente alto en los equinos. Las razones de esto no están totalmente claras, pero probablemente se refieren, al menos en parte, a los efectos de la depresión cardiorrespiratoria marcada que se produce durante la anestesia en esta especie (Muir y Hubbell, 2009). Diferentes estudios retrospectivos, prospectivos y multicéntricos que investigan los efectos adversos asociados a la anestesia y los diversos factores que influyen en el resultado de la anestesia equina sugieren que los caballos son 10 veces más propensos a sufrir una fatalidad asociada a la anestesia que los perros y gatos ( $>1$ en 100 frente a 1 en 1000) y de 5000 a 8000 veces más probabilidades de morir a causa de la anestesia que los seres humanos ( 1 en 100 frente a 1 en 500.000 a 800.000). Otro estudio multicéntrico informó que la tasa de mortalidad asociada a la anestesia de pacientes equinos y no relacionados a cólicos fue de un $0.9 \%$ (Dugdale y col,2016), esto se debe considerar, ya que los pacientes equinos que ingresan a cirugía están relativamente sanos de forma sistémica, salvo en los casos de cólico (Taylor y Clarke, 2007; Wagner, 2008; Muir y Hubbell, 2009). Los principales factores de riesgo asociados a la anestesia en equinos son:

5.1.- Edad: a mayor edad del paciente, mayor es el riesgo, los caballos sobre 14 años de edad corren un mayor riesgo de mortalidad. Así mismo, caballos de edad avanzada pueden ser más propensos a fractura de algún hueso largo en el período de recuperación, lo que resulta en eutanasia. Por otra parte, los potrillos tienen mayor riesgo de muerte y esto podría estar asociado a la falta de familiaridad con la anestesia neonatal, y la presencia de enfermedades sistémicas (Johnston y col, 1995; Johnston y col, 2002; Doherty y Valverde, 2006).

5.2.- Tipo de cirugía: la mayoría de las cirugías presentan un riesgo similar de muerte, sin embargo, complicaciones durante la recuperación anestésica incrementan las probabilidades de riesgo de volver a fracturarse que resultan en eutanasia. Largos periodos de anestesia en cirugías de reparación de fracturas también se han asociado a una mayor mortalidad. Finalmente, cirugías de emergencia (no de cólico) tienen un mayor riesgo de mortalidad (4.25 veces mayor) en comparación a una cirugía electiva y programada. En una cirugía por cólicos el riesgo de mortalidad es de un $19.5 \%$ (Johnston y col, 1995; Johnston 2004; Doherty y Valverde, 2006).

5.3.- Hora del día: procedimientos anestésicos fuera del horario normal de trabajo (generalmente entre las 18 y 20 horas o entre medianoche y 6:00 AM) conllevan un mayor riesgo para los equinos (mayor mortalidad). Esto está dado principalmente porque en general serían cirugías de urgencia, en donde todo se va realizando sobre la marcha, la preparación del paciente, la llegada del cirujano y el ingreso del paciente a pabellón demoran más tiempo, y es un periodo en el cual el paciente puede estar empeorando clínicamente, y esto asociado a el cansancio de los médicos a cargo del procedimiento después de una jornada laboral larga (Johnston y col, 1995; Johnston y col, 2002; Doherty y Valverde, 2006). 
5.4.- Elección de fármacos: el uso de anestesia inhalatoria en potrillos ( $<12$ meses de edad), sin realizar una premedicación previa, conlleva un mayor riesgo. El uso de halotano puede tener un mayor riesgo que los anestésicos inhalatorios más actuales, debido a la sensibilización que genera en el miocardio a las catecolaminas circulantes. Siguiendo una vía similar, el no usar premedicación está asociado a un riesgo mayor por el aumento de catecolaminas circulantes, las cuales se liberan por estrés. El uso de acepromacina en la premedicación disminuye el riesgo de mortalidad, haciendo al corazón menos susceptible a las arritmias ventriculares. Finalmente, la anestesia intravenosa total (TIVA) está asociada a un menor riesgo de mortalidad, pero se cree que puede ser debido a que su uso se limita a procedimientos de corta duración (Johnston y col, 1995; Johnston y col, 2002; Johnston y col, 2004; Doherty y Valverde, 2006).

5.5.- Duración de la anestesia: largos periodos anestésicos, especialmente los procedimientos que duran sobre 4 horas, con mantención por anestesia inhalatoria están a menudo asociados con depresión cardiovascular y mala perfusión tisular, por ende, con un mayor riesgo de muerte perioperatoria. Esto principalmente debido al importante efecto vasodilatador que tienen los anestésicos inhalatorios, los cuales pueden llevar a una hipotensión severa, un aumento en la frecuencia cardíaca y una disminución del gasto cardiaco, por lo cual el sistema al creer que tiene poco volumen, va a privilegiar su distribución a los órganos blanco (Johnston y col, 1995; Doherty y Valverde, 2006).

5.6.-Clasificación ASA.

Este sistema no clasifica riesgos anestésicos como tales, pero si permite una estandarización de los pacientes según historia clínica, examen físico y exámenes de laboratorio lo que permite ubicarlos dentro de alguna de las categorías del sistema de clasificación ASA, el cual ayuda a determinar la condición general del paciente y por ende las consideraciones anestésicas que se deberían tener (Cuadro 1).

Según Doherty y Valverde (2006) estas clasificaciones no son tan útiles en medicina equina, sin embargo, el sistema sirve como una guía (Tabla 1 ).

Tabla 1. Sistema de clasificación ASA (American Society of Anesthesiologists; Doherty y Valverde, 2006; Muir y Hubbell, 2009).

\begin{tabular}{|c|c|c|}
\hline $\begin{array}{c}\text { Clasificación } \\
\text { ASA }\end{array}$ & Definición & Ejemplos, no limitado a: \\
\hline ASA I & Paciente normal y saludable & - \\
\hline ASA II & $\begin{array}{c}\text { Paciente con enfermedad } \\
\text { sistémica leve }\end{array}$ & $\begin{array}{l}\text { Enfermedades leves únicas, sin } \\
\text { limitaciones funcionales sustanciales. } \\
\text { Anemia leve, Obstrucción leve y } \\
\text { recurrente de las vías aéreas. }\end{array}$ \\
\hline ASA III & $\begin{array}{l}\text { Paciente con enfermedad } \\
\text { sistémica severa }\end{array}$ & $\begin{array}{c}\text { Limitaciones funcionales sustantivas; } \\
\text { Una o más enfermedades moderadas a } \\
\text { severas. Obstrucción respiratoria } \\
\text { recurrente y severa. }\end{array}$ \\
\hline ASA IV & Paciente con enfermedad & Sepsis; Isquemia cardiaca; Disfunción \\
\hline
\end{tabular}




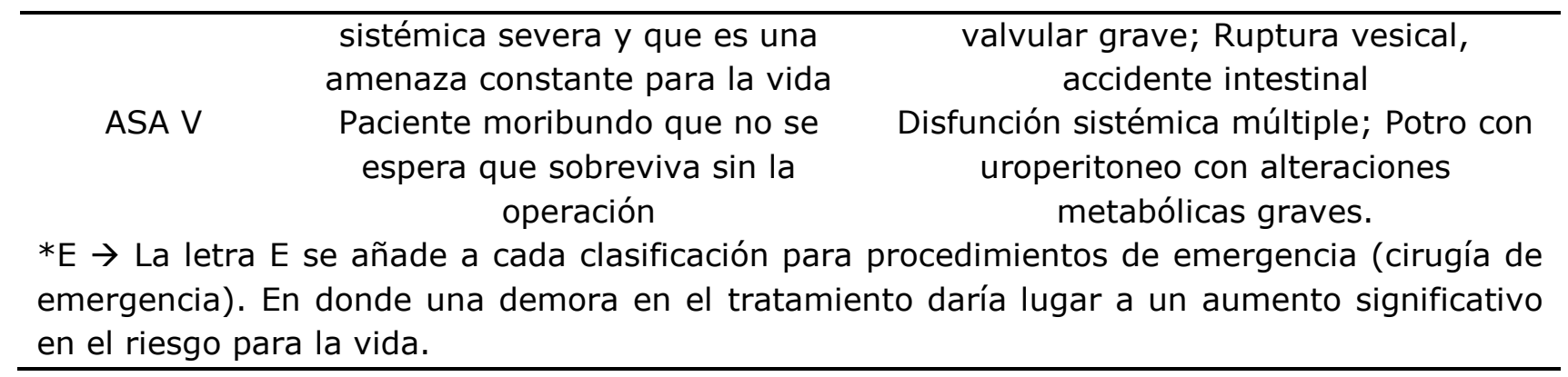

\section{6.- PROTOCOLOS ANESTÉSICOS}

Existen diversos protocolos para la inducción y mantención anestésica. Que protocolo usar dependerá de la clasificación ASA, tipo de anestesia que se utilizará y procedimiento quirúrgico al que será sometido el paciente (Doherty y Valverde, 2006; Taylor y Clarke, 2007; Muir y Hubbell, 2009). Es importante considerar que lo que se busca al anestesiar un paciente es lograr: Hipnosis, relajación muscular, analgesia y disminución del estrés, además de disminuir la probabilidad de manifestación de otros efectos indeseados (Muir y Hubbell, 2009).

Hoy en día, lo ideal es realizar una anestesia balanceada la cual es una combinación del uso de anestésico inhalatorio más anestésico intravenoso (PIVA). Esto ayudara a disminuir la CAM requerida por los anestésicos inhalatorios y también nos puede ayudar a contrarrestar algunos efectos secundarios de los fármacos que utilizamos (Doherty y Valverde, 2006, Gozalo-Marcilla y col, 2014).

Protocolo 1:

$\checkmark$ Premedicación: Xilacina 1 a $1.5 \mathrm{mg} / \mathrm{kg} / \mathrm{EV}+$ Ketamina 2 a $2.5 \mathrm{mg} / \mathrm{kg} / \mathrm{EV}$

$\checkmark$ Mantención: Isoflorano + oxígeno + PIVA (Ketamina $3 \mathrm{mg} / \mathrm{kg} / \mathrm{hr}$ )

$\checkmark$ En este caso, el uso de ketamina reduciría en aproximadamente un 30\% la CAM (Doherty y Valverde, 2006).

Protocolo 2:

$\checkmark$ Premedicación: Xilacina $1 \mathrm{mg} / \mathrm{kg} / \mathrm{EV}$ + benzodiacepina 0.02 a $0.1 \mathrm{mg} / \mathrm{kg} / \mathrm{EV}+$ Ketamina 2 a $2.5 \mathrm{mg} / \mathrm{kg} / \mathrm{EV}$

$\checkmark$ Mantención: Isoflorano + oxígeno + PIVA (Lidocaína 3-6 mg/kg/hr.)

$\checkmark$ En este caso, la lidocaína podría generar una disminución de la CAM entre un $25 \%$ a un $50 \%$, ya que es dosis dependiente (Doherty y Valverde, 2006).

$\checkmark$ Se debe cortar infusión de lidocaína unos 30 minutos antes de terminar la cirugía para que no genere problemas en la recuperación anestésica del paciente.

Protocolo 3:

$\checkmark$ Premedicación: Acepromacina 0.03 a $0.04 \mathrm{mg} / \mathrm{kg}+$ Xilacina $1 \mathrm{mg} / \mathrm{kg}+$ Ketamina 2 a $2.5 \mathrm{mg} / \mathrm{kg} / \mathrm{EV}$

$\checkmark$ Mantención: Isoflorano + oxígeno + PIVA (Lidocaína 4 mg/kg/hr + Ketamina 3 $\mathrm{mg} / \mathrm{kg} / \mathrm{hr}+$ xilacina $1 \mathrm{mg} / \mathrm{kg} / \mathrm{hr}$ )

$\checkmark$ En este caso, el uso combinado de lidocaína, ketamina y xilacina pueden llegar a reducir en un 80 - 90\% los requerimientos de CAM y pueden ser combinados para administrar mediante infusión continua (Doherty y Valverde, 2006).

$\checkmark$ Se debe cortar infusión de lidocaína unos 30 minutos antes de terminar la cirugía para que no genere problemas en la recuperación anestésica del paciente. 


\section{7.- MONITORIZACIÓN DURANTE LA ANESTESIA}

Monitorización significa vigilancia continua del paciente anestesiado. Se realiza para asegurar que la función fisiológica y la profundidad anestésica alcanzada sean las adecuadas ya que el control de la conciencia y la "profundidad de la anestesia" es la clave para una práctica anestésica segura. Por otra parte, los sistemas respiratorio y cardiovascular deben ser monitoreados de cerca. Las mediciones que se pueden hacer fácilmente, como el pulso y la frecuencia respiratoria, se utilizan para inferir información sobre la función fisiológica de los sistemas cardiovascular y respiratorio (Taylor y Clarke, 2007; Muir y Hubbell, 2009).

En la práctica diaria, dentro de las principales funciones esta la monitorización cardiovascular en los caballos anestesiados, que generalmente consiste en la evaluación clínica, electrocardiografía, oximetría de pulso y monitoreo de presiones arteriales (PAS-PAM-PAD) (Schauvliege y Gasthuys, 2013).

7.1.- Presión arterial.

La medición de presiones proporciona la información más importante sobre la función cardiovascular. La presión arterial es un indicador de la profundidad de la anestesia, ya que la hipotensión inducida por la vasodilatación que genera el agente volátil es dependiente de la dosis. Al someter a un paciente a anestesia inhalatoria, lo que se busca es mantener la presión arterial media (PAM) por encima de $70 \mathrm{~mm} \mathrm{Hg}$, esto, pensando en que la presión intracompartimental en los músculos de los caballos adultos, sobre una superficie adecuadamente acolchada, alcanza valores de 30 a 40 $\mathrm{mmHg}$ mientras que la presión transmural vascular debe ser mayor de $30 \mathrm{mmHg}$ para una adecuada microcirculación. Por esto el objetivo durante la anestesia es mantener la perfusión adecuada a todos los tejidos y la perfusión depende en gran parte del gasto cardíaco. Como es difícil poder medir el gasto cardíaco durante la anestesia clínica, se usan otros métodos para determinar de manera indirecta, el estado de la perfusión tisular (Taylor y Clarke, 2007; Schauvliege y Gasthuys, 2013), es decir, se utiliza la presión arterial (sin embargo, gasto cardíaco $=$ débito sistólico $*$ frec cardíaca, mientras que presión arterial $=$ gasto cardíaco por resistencia vascular sistémica, si un flujo $x=$ presión 1 - presión $2 /$ resistencia, vemos que la presión arterial realmente es un mal indicador de gasto cardíaco, dado que la mitad de su cálculo está basado en resistencia (vascular sistémica), sin embargo es el estimador más fácil de medir y fiable, mencionar esto en lo posible).

Dentro de los métodos de medición indirecta encontramos dos técnicas, el método Doppler y el método Oscilométrico, siendo el segundo el más utilizado en pabellón. El método oscilométrico detecta cambios en la presión interior del manguito (causada por la onda de pulso) a partir de la cual se calculan la presión sistólica, diastólica, la presión media y la frecuencia cardíaca, la anchura del manguito debe ser del $40 \%$ de la circunferencia de la extremidad/cola, por lo que generalmente los errores de lectura resultan del tamaño incorrecto del manguito (Doherty y Valverde, 2006). El más utilizado en pabellón es el método directo invasivo, es decir con catéter intra arterial y transductor de presión y es el gold-standard en equinos.

7.2.- Pulso y Oximetría de pulso.

El pulso debe ser palpado regularmente durante la anestesia y en el paciente equino es bien fácil poder tomarla ya que está provisto de varias arterias periféricas superficiales: las faciales, las faciales transversales, los grandes metatarsianos, las 
arterias digitales o digitales comunes son de fácil acceso. Se pueden utilizar los monitores de pulso como el sistema Doppler en la cola para indicar la frecuencia y la calidad del pulso. La oximetría de pulso también actúa como monitor de pulso periférico. Lo importante de tomar el pulso es que la calidad del pulso proporciona información importante sobre la circulación periférica e indirectamente, sobre la contractilidad cardíaca pero no indica la presión arterial. Sin embargo, está relacionado con el volumen sistólico y un pulso débil generalmente se asocia con hipotensión. El pulso también se siente débil cuando hay una vasoconstricción intensa, tal como ocurre después de que se ha administrado un agonista a2, aunque en estos casos la presión sanguínea es alta (Doherty y Valverde, 2006; Taylor y Clarke, 2007).

Los oxímetros de pulso muestran la frecuencia cardíaca y se utilizan para obtener o estimar indirectamente el porcentaje de hemoglobina en sangre arterial que se encuentra saturada con oxígeno $\left(\mathrm{SaO}_{2}\right)$. Su función depende de un pulso periférico razonable para producir una medición (valor de $\mathrm{SpO}_{2}$ ) y por lo tanto servir como un índice de la perfusión tisular (Muir y Hubbell, 2009).

\section{3.- Membranas mucosas.}

El color de las mucosas y el tiempo de rellene capilar son una guía para ver la oxigenación y la adecuada perfusión periférica. El mejor sitio para evaluar en el caballo es en la boca. El tiempo de rellene capilar de 2 o más segundos es motivo de alerta, ya que está asociado con una perfusión inadecuada. El color de las mucosas siempre es un parámetro más subjetivo, pero se determina que, las membranas mucosas deben ser rosadas. Membranas mucosas claras con un lento tiempo de rellene capilar y un ligero tinte gris, y que a menudo se ha visto en pacientes en los cuales se está usando halotano, presumiblemente indican una perfusión periférica menor a la adecuada. Un buen color rosa saludable puede indicar hipercapnia, pero también indica buena perfusión, etc. Si se observa una mejora en el color de las mucosas es un signo de respuesta adecuada al tratamiento y de una mejor perfusión periférica (Doherty y Valverde, 2006; Taylor y Clarke, 2007).

7.4.- Electrocardiograma (ECG).

Este tipo de herramienta nos entrega información sobre la actividad eléctrica del corazón. Se debe tener en consideración que el ritmo normal no implica necesariamente que exista un gasto cardiaco adecuado. Los cambios en la configuración del ritmo pueden estar asociados con anomalías circulatorias. Es esencial para cualquier tratamiento que sea necesario instaurar que se identifique la naturaleza de cualquier arritmia. Los cambios en la configuración del ECG pueden indicar anomalías sistémicas: por ejemplo, la onda $T$ puede cambiar con hipoxemia o alteraciones electrolíticas. La fibrilación auricular se desarrolla ocasionalmente durante la anestesia y no se puede diagnosticar sin ECG (Doherty y Valverde, 2006; Taylor y Clarke, 2007; Muir y Hubbell, 2009).

7.5.- Capnografía.

El dióxido de carbono $\left(\mathrm{CO}_{2}\right)$ es un subproducto del metabolismo del tejido, transportado a los pulmones por la sangre que vuelve al corazón, y exhalado en la atmósfera. Capnografía es la medición completa y la visualización de la forma de onda de $\mathrm{CO}_{2}$ espirado en tiempo real. Las presiones parciales ensiones de dióxido de 
carbono (usar CO2) exhalado se pueden analizar continuamente. Teóricamente, la tensión de $\mathrm{CO}_{2}$ dentro del tubo endotraqueal al final de la espiración debe estar en equilibrio con la tensión de $\mathrm{CO}_{2}$ en la sangre que sale de los alvéolos (la sangre arterial) (por el mismo fenómeno que el ETiso es = al ET cerebral). Por lo tanto, mediante la medición de $\mathrm{ETCO}_{2}$ (End-tidal $\mathrm{CO}_{2}$ ), se puede inferir información sobre la tensión sanguínea arterial $\mathrm{CO}_{2}\left(\mathrm{PaCO}_{2}\right)$. El $\mathrm{ETCO}_{2}$ se utiliza para evaluar la ventilación (hipoventilación, hiperventilación), verificar la correcta posición del tubo endotraqueal, asegurar la vía aérea y la integridad del circuito anestésico/ventilador (Doherty y Valverde, 2006; Muir y Hubbell, 2009).

7.6.- Plano anestésico.

Para todo procedimiento que se llevara a cabo utilizando anestesia inhalatoria, es necesario lograr obtener un plano anestésico adecuado. El nivel de conciencia o profundidad anestésica, es una preocupación clave durante la anestesia en equinos. Los pacientes que están muy superficiales en su plano pueden responder a la estimulación quirúrgica, lo que resulta en un estrés excesivo, el movimiento y la contaminación del sitio quirúrgico y las lesiones a sí mismos y al personal de quirófano. Por otra parte, los pacientes que están en un plano anestésico muy profundo pueden sufrir una depresión cardiorrespiratoria excesiva, predisponiendo a una recuperación deficiente y miopatía. Históricamente, la profundidad de la anestesia se ha categorizado en etapas y planos tanto para fines descriptivos como académicos (Tabla 2) (Muir y Hubbell, 2009).

Tabla 2. Monitorización de la profundidad anestésica: modificado para caballos de etapas y planos de anestesia de Guedel (Muir y Hubbell, 2009).

\begin{tabular}{|c|c|c|c|}
\hline Etapa & $\begin{array}{l}\text { Posición/tamaño de } \\
\text { pupila }\end{array}$ & Reflejos de ojos & $\begin{array}{l}\text { F. Cardiaca/F.Resp. } \\
\text { /Presión arterial }\end{array}$ \\
\hline 1 (Analgesia) & Central/Pequeña & P/C presente & Aumento variable \\
\hline 2 (Delirio) & Central(activo)/Grande & $\mathrm{P} / \mathrm{C}$ presente & Aumento variable \\
\hline 3 - Plano 1 (Leve) & Ventromedial/Pequeña & $\begin{array}{c}\text { P levemente } \downarrow \\
\text { C presente }\end{array}$ & Normal o elevado \\
\hline 3 - Plano 2 (Moderado) & Ventromedial/mediana & $\begin{array}{l}\text { P disminuido } \\
\text { C levemente } \downarrow\end{array}$ & Normal o levemente $\downarrow$ \\
\hline $\begin{array}{c}3 \text { - Plano } 3 \text { (Moderado } \\
\text { - Profundo) }\end{array}$ & Central/mediana & $\mathrm{P} / \mathrm{C}$ disminuido & $\begin{array}{c}\text { Disminución mínima a } \\
\text { moderada }\end{array}$ \\
\hline 3 - Plano 4 (Profundo) & Central/grande & $\begin{array}{l}\text { P ausente } \\
\text { C muy } \downarrow\end{array}$ & $\begin{array}{l}\text { Marcadamente } \\
\text { disminuidos }\end{array}$ \\
\hline $\begin{array}{c}4 \text { (Sobredosis) } \\
* \text { P } \rightarrow \text { Reflejo palpebral; } \\
* \downarrow \rightarrow \text { disminuido }\end{array}$ & $\begin{array}{l}\text { Central y muy dilatada } \\
C \rightarrow \text { Reflejo corneal }\end{array}$ & $\mathrm{P} / \mathrm{C}$ ausentes & Colapso \\
\hline
\end{tabular}

Finalmente, el objetivo de la monitorización es asegurar que la función fisiológica y la profundidad de la anestesia son adecuadas, para ello se debe mantener un registro de las mediciones realizadas durante la anestesia mediante el uso de una ficha anestésica. Esto permite ver las tendencias durante el tiempo anestésico y, en caso de que ocurra algún problema, proporciona un registro de lo sucedido y realizado. La vigilancia, conciencia y respuesta oportuna a los signos clínicos es particularmente 
importante en equinos porque el masaje cardíaco externo es muy limitado y las terapias de reanimación también son limitadas.

\section{8.- COMPLICACIONES DURANTE LA ANESTESIA}

Durante los procedimientos que conllevan un tiempo anestésico variable, se pueden presentar diferentes complicaciones, las cuales pueden ser de mayor o menor riesgo para la salud del paciente, estas se pueden presentar en cualquier momento de los tiempos anestésicos asociados. Según lo descrito por diferentes autores, dentro de las complicaciones más frecuentes podemos encontrar:

8.1.- Hipotensión: complicación más común que se produce por el uso de anestésicos inhalatorios. Los equinos son una de las especies particularmente susceptibles a los efectos vasodilatadores que tiene este tipo de anestesia. Se ha demostrado que una presión arterial media (PAM) de $60 \mathrm{mmHg}$ o menos durante la anestesia aumenta el riesgo de miopatía pos anestésica. Por lo tanto, se debe tratar de mantener la PAM en $70 \mathrm{mmHg}$ o más en pacientes equinos anestesiados. Hay varias formas para aumentar la PAM en animales hipotensos: (1) Disminuir la entrega de anestésico inhalatorio u otros agentes potencialmente hipotensores; (2) aumentar la administración de fluidos por vía intravenosa; (3) administrar fármacos inotrópicos; y (4) administrar vasopresores (Doherty y Valverde, 2006; Taylor y Clarke, 2007; Wagner, 2008).

8.2.- Hipercapnia: anestésicos inhalatorios poseen efectos depresores sobre el sistema respiratorio y los equinos son más susceptibles a estos, esto en conjunto a la relajación muscular y la recumbencia prolongada pueden, con frecuencia, conducir a una hipoventilación con un aumento de la $\mathrm{PCO}_{2}$ (Hipercapnia) (Doherty y Valverde, 2006; Taylor y Clarke, 2007; Wagner, 2008).

8.3.- Hipoxemia: en los equinos se estima que una saturación de $90 \%$ se obtiene con una $\mathrm{PO}_{2}$ de $54 \mathrm{mmHg}$ lo que significa que, en comparación con los humanos y los perros, los caballos son un poco más tolerantes a valores bajos de $\mathrm{PO}_{2}$, por lo cual, una hipoxemia es más probable que ocurra en pacientes con anestesia inyectable sin suplementación de oxigeno con una $\mathrm{PO}_{2}$ arterial menor a $60 \mathrm{mmHg}$. En un paciente sometido a anestesia inhalatoria es muy poco frecuente que pueda padecer de una hipoxemia absoluta ya que en este caso están con un flujo de oxigeno constante . La recumbencia dorsal y la distensión abdominal son factores que pueden aumentar la probabilidad de hipoxemia en estos pacientes (Doherty y Valverde, 2006; Taylor y Clarke, 2007; Wagner, 2008).

8.4.- Alteración de frecuencia cardiaca o en el ritmo cardiaco: la frecuencia cardíaca en los equinos anestesiados no varía mucho con el plano anestésico alcanzado o con la estimulación quirúrgica, por lo que no es fiable como un indicador de una profundidad adecuada, a diferencia de otras especies. Dentro de las alteraciones que podemos encontrar, las más frecuentes son:

8.4.1.- Bradicardia: el paciente presenta una frecuencia cardíaca < a 25 latidos por minuto. Esto generalmente está asociado con la administración de fármacos específicos, como alfa-2 agonistas o dobutamina. Si tenemos una frecuencia cardíaca que permanece mayor a 20 latidos por minuto y la PAM se mantiene igual o mayor a $70 \mathrm{mmHg}$, generalmente no se requiere tratamiento específico para manejar la bradicardia. Sin embargo, si la frecuencia cardíaca es inferior a 20 latidos por minuto, 
se puede usar una dosis de atropina a 0,002-0,003 mg/kg por vía intravenosa, la cual suele ser suficiente para restablecer la frecuencia cardíaca de al menos 25 latidos por minuto, sin causar efectos apreciables sobre la motilidad gastrointestinal (Doherty y Valverde, 2006; Taylor y Clarke, 2007; Wagner, 2008).

8.4.2.- Taquicardia: la presencia de frecuencias cardíacas $>$ a 50 latidos por minuto es bastante inusual en los equinos anestesiados, y no suele indicar una profundidad anestésica insuficiente. De hecho, la frecuencia cardíaca tiende a aumentar ligeramente, aunque no significativamente, con los planos anestésicos más profundos con sevoflorano. La taquicardia durante la anestesia general puede resultar de una estimulación simpática preexistente, como ocurre en algunos casos de cólico, o puede desarrollarse intraquirúrgicamente a partir de una hipoxemia o el uso de un torniquete. Los fármacos inotrópos, como la dobutamina o la dopamina, pueden en ocasiones causar o exacerbar la taquicardia en algunos caballos, por lo que en esos casos se recomienda terminar o reducir la tasa de infusión y se puede corregir la taquicardia en algunos pacientes. Se debe descartar o corregir anormalidades en el volumen vascular y los gases en sangre $\left(\mathrm{PCO}_{2}, \mathrm{PO}_{2}\right)$, así como evaluar la profundidad anestésica. Si la taquicardia persiste a pesar de la corrección de anormalidades, se puede utilizar una dosis de alfa-2 agonista a 0.1-0.2 mg/kg de xilacina, por ejemplo, la cual puede ayudar a bajar la frecuencia cardíaca (Doherty y Valverde, 2006; Taylor y Clarke, 2007; Wagner, 2008).

8.4.3.- Bloqueo atrioventricular de 20: es una arritmia común en los caballos despiertos y no sedados y se puede esperar que se produzca o se exacerbe después de la administración de un alfa-2 agonistas. Si una dosis preanestésica de alfa-2 agonista causa un bloqueo atrioventricular de segundo grado, al realizar la inducción con ketamina o tiopental aumentará la frecuencia cardíaca y eliminará el bloqueo. Los pacientes que han recibido altas dosis de alfa-2 agonistas, o que están con una infusión constante de alfa-2 agonistas, o que están recibiendo dobutamina durante la anestesia inhalatoria, son más propensos a presentar un bloqueo de este tipo. En general, el bloqueo atrioventricular de segundo grado en sí no genera problemas, siempre y cuando la frecuencia cardiaca no sea demasiado baja ( $<20$ latidos por minuto). En esos casos se aplican las mismas medidas que para el tratamiento de la bradicardia (Doherty y Valverde, 2006; Taylor y Clarke, 2007; Wagner, 2008).

8.4.4.- Contracción ventricular prematura (CVP): a diferencia de los perros, los equinos raramente experimentan contracciones ventriculares prematuras durante la anestesia. Cuando ocurren en un equino anestesiado, hay mayor preocupación ya que estas en caballos pueden progresar a taquicardia ventricular o fibrilación ventricular con menos advertencia que en otras especies. Si se está usando halotano, se debe cambiar a isoflorano o sevoflorano, ya que esto puede reducir la frecuencia de las CVPs. Se debe asegurar una ventilación adecuada $\left(\mathrm{PCO}_{2}\right)$, la oxigenación $\left(\mathrm{PO}_{2}\right)$ y la profundidad de la anestesia en estos pacientes. La presencia de CVPs se puede manejar con la administración de lidocaína a $0.5-1.5 \mathrm{mg} / \mathrm{kg}$ en un bolo para control inmediato o una infusión constante a dosis de $25-50 \mathrm{mg} / \mathrm{kg} / \mathrm{min}$ si es que persisten las CVPs (Doherty y Valverde, 2006; Taylor y Clarke, 2007; Wagner, 2008).

8.4.5.- Fibrilación atrial: La mayoría de los pacientes con fibrilación atrial toleran la anestesia razonablemente bien, pueden tender más a la hipotensión que los equinos con ritmo normal, pero la fibrilación atrial no es necesariamente una contraindicación 
para la anestesia. Incluso, en muchos casos la anestesia general es necesaria para algunos tipos de electrocardioversión de la fibrilación atrial. En estos casos se recomienda minimizar el uso de fármacos alfa-2 agonistas, utilizar acepromacina para la sedación y evitar el uso de tiopental y halotano (Doherty y Valverde, 2006; Taylor y Clarke, 2007; Wagner, 2008).

8.5.- Hipertensión: es muy poco frecuente que ocurra en pacientes sometidos a anestesia inhalatoria, ya que lo que generalmente ocurre es una hipotensión. La hipertensión se podría dar en procedimientos que son especialmente dolorosos como lo es una neurectomía. También se podría dar en casos de hipoxemia o hipercapnia grave (Doherty y Valverde, 2006; Taylor y Clarke, 2007; Wagner, 2008).

\section{9.- COMPLICACIONES POS ANESTESICAS}

El momento de recuperación anestésica corresponde a uno de los tiempos más complicados luego de cualquier procedimiento anestésico, en el cual se pueden presentar diversas complicaciones, según la descripción de algunos autores (Doherty y Valverde, 2006; Taylor y Clarke, 2007; Wagner, 2008) dentro de estas podemos encontrar:

9.1.- Fracturas o lesiones de tejidos blandos: no son comunes, son una de las complicaciones más complejas de la anestesia equina. Algunos estudios retrospectivos informan que los huesos fracturados ocurren en aproximadamente el 0,2\% de los caballos anestesiados ( 2 de cada 1000). La evidencia clínica sugiere que tales fracturas ocurren a menudo en o cerca del sitio de una lesión preexistente. Pacientes de edad más avanzada también pueden estar en mayor riesgo. Por lo que se recomienda que la recuperación sea asistida o controlada para minimizar el riesgo de fracturas, sobre todo en pacientes con historial de fracturas o pacientes muy debilitados. En estos casos donde se sabe que algún miembro sufrió una fractura, se puede usar un yeso o un vendaje sólo para el proceso de recuperación y así minimizar la tensión en la reparación de la fractura.

Es frecuente encontrarse con lesiones de tejidos blandos, como laceraciones de los labios, lengua o extremidades, pero rara vez son graves. Los caballos anestesiados también corren riesgo de abrasión corneal durante la anestesia y la recuperación, por lo que se debe tener especial cuidado para proteger los ojos del caballo.

9.2.- Miopatía: Las localizaciones típicas son el macetero, tríceps, glúteos y sobre las costillas. Una forma de poder evitar esto es poner más relleno en estas áreas, lo cual puede reducir el problema. Si se produce miopatía local o generalizada después de la anestesia, se recomienda administrar AINES, como la fenilbutazona (4 $\mathrm{mg} / \mathrm{kg} / \mathrm{EV}$ ), fluidoterapia para promover la diuresis y ayudar a la eliminación de la mioglobina y en ocasiones se puede usar acepromacina $(0,02$ a 0,03 mg/Kg/EV) para promover la vasodilatación y mejorar la perfusión muscular.

9.3.- Neuropatía: Los nervios superficiales, como los nervios facial y radial, están sujetos a daño por presión durante la anestesia y la posición en decúbito, o por un estiramiento o tensión excesiva de un nervio. Se debe proporcionar cuidadosamente un buen relleno para proteger estos nervios de tal daño por compresión. El nervio facial está particularmente en riesgo durante las cirugías que afectan a la cabeza o al ojo, y en donde se someta la cabeza a un peso o presión extra. El nervio radial es el que se encuentra más vulnerable estando el paciente en 
decúbito lateral, generalmente ocurre por un problema de relleno insuficiente, o por que las almohadillas no apoyan completamente la región del hombro y del codo.

9.4.- Edema pulmonar: Se han reportado casos de edema pulmonar posanestésico en caballos en asociación con obstrucción transitoria de las vías respiratorias o re expansión del pulmón que estuviera con atelectasia durante la anestesia. En estos pacientes podemos observar signos clínicos iniciales asociados a edema pulmonar tales como, taquipnea, taquicardia, hipoxemia e hipercapnia. Como manejo medico se recomienda oxigenoterapia y uso de algún diurético, como furosemida a 0,5 - $1 \mathrm{mg} / \mathrm{kg} / \mathrm{EV}$. También se recomienda el uso de AINES, fenilbutazona a $4 \mathrm{mg} / \mathrm{kg} /$ o de un antiinflamatorio esteroidal como la dexametasona a 0,02-0,04 $\mathrm{mg} / \mathrm{kg} / \mathrm{EV}$ para disminuir cualquier potencial inflamación de las vías respiratorias.

9.5.- Tiempo de recuperación prolongado: en general las recuperaciones de la anestesia inhalatoria demoran menos de 1 hora. Los anestésicos inhalatorios como el isoflorano se eliminan rápidamente del cerebro a la sangre y luego a los pulmones, donde se exhalan. Sin embargo, en los casos donde se han administrado otros fármacos, como alfa-2 agonistas, poco antes del final de la anestesia, o durante la anestesia, muy probablemente la recuperación se prolongue de una manera dependiente a la dosis administrada. Un paciente que esté tomando más tiempo de lo esperado para recuperarse debe ser evaluado completamente para ver si es que existe alguna otra razón clínica que este demorando el proceso, como por ejemplo una lesión musculo esquelética, como fractura o miopatía; algunas anomalías metabólicas, como hipoglicemia o hipocalcemia.

\section{CONCLUSIONES}

Ningún procedimiento anestésico está exento de riesgos, ya sea en medicina humana como en medicina veterinaria, estos pueden afectar tanto la salud y el bienestar del animal como también la salud e integridad física del personal presente en estos casos. Es por esto, que se debe tener en consideración todas aquellas variables que nos pueden ayudar a disminuir al máximo estos riesgos.

El objetivo fundamental de la anestesia inhalatoria es lograr inmovilizar al caballo, permitir aliviar el dolor y ayudar a proporcionar las condiciones óptimas para cirugías u otros procedimientos que estén relacionados con mejorar la salud del paciente que lo requiere. Para poder lograr un plano anestésico adecuado y que se mantenga durante el tiempo requerido para dicho procedimiento, es necesario lograr y mantener una concentración óptima de anestésico en el SNC y esto se puede lograr mediante el uso de una anestesia balanceada.

Para lograr una adecuada mantención con anestesia inhalatoria es necesario realizar una buena premedicación, según la condición general del paciente y así poder lograr una buena inducción, que es uno de los factores que contribuirán a disminuir los riegos de golpes y lesiones asociadas al derribo, tener al equino sometido a un menor tiempo anestésico y así tratar de disminuir al máximo las complicaciones asociadas al uso prolongado de anestésicos inhalatorios. Además, se pueden disminuir los riesgos 
anestésicos realizando una adecuada clasificación del estado de salud general del animal.

Es nuestra labor estar monitoreando constantemente al paciente que se encuentra anestesiado, medir frecuentemente presiones, y chequear el pulso, ya que queremos evitar al máximo la presentación de hipotensión marcada u otras complicaciones.

La evaluación de los equipos de anestesia y de los circuitos respiratorios son fundamentales para garantizar la seguridad del personal y de los pacientes. Para los pacientes, el suministro de concentraciones y cantidades adecuadas de oxígeno y anestésicos es esencial. Para el personal, la máquina y el circuito deben mantenerse para evitar la contaminación del lugar de trabajo por la fuga de anestésicos.

La morbilidad y mortalidad en la anestesia equina clínica es considerablemente mayor que en otras especies domésticas y esto suele ser causado por hipotensión e hipoventilación durante la anestesia. Todos los agentes anestésicos volátiles causan depresión cardiopulmonar dependiente de la dosis. Además, los caballos posicionados en decúbito dorsal caen en una depresión cardiovascular más severa que aquellos situados en decúbito lateral.

Finalmente, el uso en conjunto de anestésicos intravenosos, analgesia y anestesia inhalatoria, se traduce en una anestesia general más efectiva y segura, en comparación a si se usará el agente inhalatorio por sí solo.

\section{REFERENCIAS}

Brosnan, RJ, 2013. Inhaled Anesthetics In Horses. Vet. Clin. North America: Equin. Pract. 29: $69-87$

Clark-Price, SC, 2013. Recovery of horses from anesthesia. Vet. Clin. North America: Equin. Pract., 29(1), 223-242.

Doherty, T., A Valverde. 2006. Manual of Equine Anesthesia and Analgesia, First Ed. Blackwell Publishing, Oxford, UK, pp. $1-351$

Driessen, B., L Nann, R Benton, R Boston. 2006. Differences in need for hemodynamic support in horses anesthetized with sevoflurane as compared to isoflurane. Vet. Anaest. Analg. 33: $356-367$.

Dugdale, A.H., J Obhrai, PJ Cripps. 2016. Twenty years later: a single-centre, repeat retrospective analysis of equine perioperative mortality and investigation of recovery quality. Vet. Anaest. Analg., 43: 171-178.

Dugdale, AH, PM Taylor. 2016. Equine anaesthesia-associated mortality: where are we now?. Vet. Anaest. Analg., 43: 242-255.

Durongphongtorn, S., WN McDonell, CL Kerr, FJT Neto, KK Mirakhur. 2006. Comparison of hemodynamic, clinicopathologic, and gastrointestinal motility effects and recovery characteristics of anesthesia with isoflurane and halothane in horses undergoing arthroscopic surgery. American J. Vet. Res, 67: 32-42. 
Fischer, B, S Clark-Price. 2015. Anesthesia Of The Equine Neonate In Health and Disease. Vet. Clin. North America: Equin. Pract. 31: 567 - 585.

Gozalo-Marcilla, M, F Gasthuys, S Schauvliege. 2014. Partial intravenous anaesthesia in the horse: a review of intravenous agents used to supplement equine inhalation anaesthesia. Part 1: lidocaine and ketamine. Vet. Anaest. Analg. 41: 335-345.

Johnston, GM, PM Taylor, MA, Holmes, JLN Wood. 1995. Confidential enquiry of perioperative equine fatalities (CEPEF-1): preliminary results. Equ. Vet. J., 27: 193-200.

Johnston, GM, JK Eastment, JLN Wood, PM Taylor. 2002. The confidential enquiry into perioperative equine fatalities (CEPEF): mortality results of Phases 1 and 2. Vet. Anaest. Analg., 29: 159-170.

Johnston, GM, JK Eastment, PM Taylor, JLN Wood. 2004. Is isoflurane safer than halothane in equine anaesthesia? Results from a prospective multicentre randomised controlled trial. Equ. Vet. J., 36: 64-71.

Kahn, CM, 2007. Manual Merck De Veterinaria. Sexta Ed. Océano, Barcelona, España, pp.

Klein, BG, 2014. Cunningham Fisiología Veterinaria, Quinta Ed. Saunders Elsevier, Barcelona, España, pp. 158 - 262; 495 - 542

Kushiro, T, K Yamashita, MA Umar, S Maehara, S Wakaiki, R Abe, T Seno, K Tsuzuki, Y Izumisawa. W Muir. 2005. Anesthetic and Cardiovascular Effects of Balanced Anesthesia Using Constant Rate Infusion of Midazolam-Ketamine-Medetomidine with Inhalation of Oxygen-Sevoflurane (MKM-OS Anesthesia) in Horses. J. Vet. Med. Sci., 67: 379-384.

Lin, HC, T Passler, RR Wilborn, JS, Taintor, FJ Caldwell, 2015. A review of the general pharmacology of ketamine and its clinical use for injectable anaesthesia in horses. Equ. Vet. Educ, 27: 146-155.

Mee, AM, PJ Cripps, RS Jones, 1998. A retrospective study of mortality associated with general anaesthesia in horses: elective procedures. Vet. Rec., 142: 275-276.

Mee, AM, PJ Cripps, RS, Jones, 1998. A retrospective study of mortality associated with general anaesthesia in horses: emergency procedures. Vet. Rec., 142, 307-308.

Mitchell, B, 1969. Equine anaesthesia: an assessment of techniques used in clinical practice. Equ. Vet. J, 1: 261-275.

Moens, Y, 2013. Mechanical Ventilation and Respiratory Mechanics During Equine Anesthesia. Vet. Clin. North America: Equin. Pract., 29: 51-67. 
Muir, WW, JAE Hubbell, 2009. Equine Anesthesia: Monitoring and Emergency Therapy, Second Ed. Saunders Elsevier, St. Louis, MO, USA, pp. 1 - 446

Nannarone, S, C Spadavecchia, 2012. Evaluation of the clinical efficacy of two partial intravenous anesthetic protocols, compared with isoflurane alone, to maintain general anesthesia in horses. American J. Vet. Res, 73: 959-967.

Raisis, AL, LE Young, KJ Blissitt, JC Brearley, HB Meire, PM Taylor, P Lekeux, 2000. A comparison of the haemodynamic effects of isoflurane and halothane anaesthesia in horses. Equ. Vet. J, 32: 318-326.

Schauvliege, S, F Gasthuys, 2013. Drugs for Cardiovascular Support in Anesthetized Horses. Vet. Clin. North America: Equin. Pract., 29: 19-49.

Senior, JM, 2013. Morbidity, mortality, and risk of general anesthesia in horses. Vet. Clin. North America: Equin. Pract., 29: 1-18.

Taylor PM, KW Clarke, 2007. Handbook of Equine Anesthesia, Second Ed. Saunders Elsevier, Philadelphia, PA, USA, pp. $1-175$

Tranquilli, WJ, JC Thurmon, KA Grimm, 2007. Lumb \& Jones' Veterinary Anesthesia and Analgesia, Fourth Ed. Blackwell Publishing, Oxford, UK, pp. 12 - 1048

Valverde, A, 2013. Balanced anesthesia and constant-rate infusions in horses. Vet. Clin. North America: Equin. Pract., 29: 89-122.

Valverde, A, C Gunkel, TJ Doherty, S Giguère, AS Pollak, 2005. Effect of a constant rate infusion of lidocaine on the quality of recovery from sevoflurane or isoflurane general anaesthesia in horses. Equ. Vet. J., 37: 559-564.

Wagner, $\mathrm{AE}, 2008$. Complications In Equine Anesthesia. Vet. Clin. North America: Equin. Pract. 24: 735 - 752. 